\title{
Symmetric stability of compressible zonal flows on a generalized equatorial $\beta$ plane
}

Article

Published Version

Fruman, M. D. and Shepherd, T. G. (2008) Symmetric stability of compressible zonal flows on a generalized equatorial $\beta$ plane. Journal of the Atmospheric Sciences, 65 (6). pp. 19271940. ISSN 0022-4928 doi:

https://doi.org/10.1175/2007JAS2582.1 Available at https://centaur.reading.ac.uk/32038/

It is advisable to refer to the publisher's version if you intend to cite from the work. See Guidance on citing.

Published version at: http://dx.doi.org/10.1175/2007JAS2582.1

To link to this article DOI: http://dx.doi.org/10.1175/2007JAS2582.1

Publisher: American Meteorological Society

All outputs in CentAUR are protected by Intellectual Property Rights law, including copyright law. Copyright and IPR is retained by the creators or other copyright holders. Terms and conditions for use of this material are defined in the End User Agreement.

\section{www.reading.ac.uk/centaur}

\section{CentAUR}

Central Archive at the University of Reading 
Reading's research outputs online 


\title{
Symmetric Stability of Compressible Zonal Flows on a Generalized Equatorial $\beta$ Plane
}

\author{
MARK D. Fruman* AND TheOdore G. ShePherd \\ Department of Physics, University of Toronto, Toronto, Ontario, Canada
}

(Manuscript received 7 August 2007, in final form 27 October 2007)

\begin{abstract}
Sufficient conditions are derived for the linear stability with respect to zonally symmetric perturbations of a steady zonal solution to the nonhydrostatic compressible Euler equations on an equatorial $\beta$ plane, including a leading order representation of the Coriolis force terms due to the poleward component of the planetary rotation vector.

A version of the energy-Casimir method of stability proof is applied: an invariant functional of the Euler equations linearized about the equilibrium zonal flow is found, and positive definiteness of the functional is shown to imply linear stability of the equilibrium. It is shown that an equilibrium is stable if the potential vorticity has the same sign as latitude and the Rayleigh centrifugal stability condition that absolute angular momentum increase toward the equator on surfaces of constant pressure is satisfied. The result generalizes earlier results for hydrostatic and incompressible systems and for systems that do not account for the nontraditional Coriolis force terms.

The stability of particular equilibrium zonal velocity, entropy, and density fields is assessed. A notable case in which the effect of the nontraditional Coriolis force is decisive is the instability of an angular momentum profile that decreases away from the equator but is flatter than quadratic in latitude, despite its satisfying both the centrifugal and convective stability conditions.
\end{abstract}

\section{Introduction}

The simplest instability of a flow in a planetary atmosphere that one might consider is that of an axisymmetric (longitude independent) equilibrium subject to axisymmetric perturbations. This is the problem of symmetric stability. When viscosity and diabatic effects are neglected, the problem may be phrased in terms of the distributions of entropy and zonal angular momentum, both materially conserved quantities, and is accordingly a generalization of convective stability to nonresting flows and of inertial instability to stratified flows. It is of particular interest for flows in the equatorial region, where zonal angular momentum has its maximum value and inertial instability is readily ob-

* Current affiliation: Laboratoire de Physique des Oceans, IFREMER, Brest, France.

Corresponding author address: T. G. Shepherd, Department of Physics, University of Toronto, 60 St. George Street, Toronto, ON M5S 1A7, Canada.

E-mail: tgs@atmosp.physics.utoronto.ca served. However, it is also in the equatorial region that the planetary rotation vector becomes tangent to the surface and the usual neglect of the effect of its tangential component is most tenuous.

When the atmosphere is shallow compared to the radius of the planet, a suite of approximations is commonly made to the governing equations, consisting of hydrostatic balance in the radial direction, the neglect of the explicit radial dependence in the Lagrangian derivative and metric terms, and the neglect of the Coriolis force terms due to the poleward component of the planetary rotation vector. The third of these, known as the "traditional approximation" after Eckart (1960), is necessitated by the desire to retain exact conservation of energy and angular momentum (Phillips 1966; Veronis 1968; Lorenz 1967) and is justifiable in many midlatitude Earth problems because of the smallness of the Coriolis parameter compared to the buoyancy frequency (see, e.g., Gill 1982). The question of its justifiability in general and especially near the equator has been raised by Veronis (1963) and more recently by White and Bromley (1995) and Colin de Verdière and Schopp (1994), who all conclude that, at least in the immediate vicinity of the equator, the approximation is 


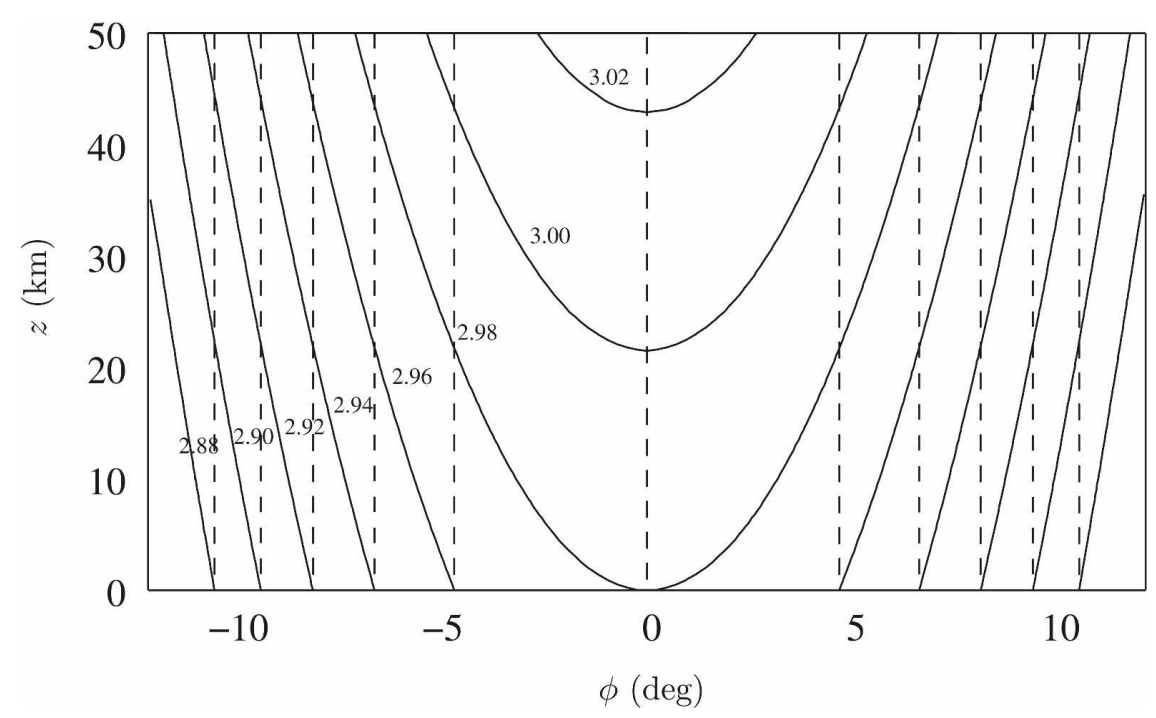

FIG. 1. Contours of constant planetary angular momentum $\Omega r^{2} \cos ^{2} \phi$ without the traditional approximation (solid lines) and with $r=a$ (dashed) as functions of latitude $\phi$ and distance above the surface $z$. Contour values are in $10^{9} \mathrm{~m}^{2} \mathrm{~s}^{-1}$. Contours that meet at the surface have the same value. The approximately parabolic contours correspond to cylinders of constant distance from the rotation axis of the spherical earth.

not obviously valid on the basis of the length and time scales of typically observed motions. Colin de Verdière and Schopp argue that, for the neglect of the nontraditional terms, the meridional length scale of the motion should be large compared to $(H a)^{1 / 2}$, where $H$ is a characteristic vertical scale of the motion and $a$ the radius of the planet. For the middle atmosphere, $(\mathrm{Ha})^{1 / 2}$ can be on the order of $5^{\circ}$ latitude. The Met Office currently uses a forecasting model based on a nontraditional "quasihydrostatic" model (White 1999). Hua et al. (1997) did not make the approximation in a study of equatorial inertial instability, and Kasahara (2003), Durran and Bretherton (2004), Thuburn et al. (2002), and Gerkema and Shrira (2005) have studied linear wave propagation in the more general system.

An immediate consequence of the traditional approximation is that curves of constant planetary angular momentum, meaning the angular momentum about the planetary rotation axis of fluid parcels at rest in the rotating frame, become lines of constant latitude, whereas in the unapproximated system they are the curves on which $r^{2} \cos ^{2} \phi$ is constant. This is illustrated in Fig. 1. As a result, when the traditional approximation is made, static and inertial stability are in a sense orthogonal: static stability requires that entropy increase with height above the surface, and inertial stability requires that zonal angular momentum increase toward the equator on surfaces of constant pressure. The latter is an expression of Rayleigh's (1917) centrifugal stability theorem. In addition, symmetric stabil- ity requires that potential vorticity have the same sign as latitude, which is to say that angular momentum must also increase toward the equator on surfaces of constant entropy (see, e.g., Stevens 1983; Bowman and Shepherd 1995).

Dunkerton (1981) addressed the simplest interesting case of inertial symmetric instability, that of uniform stable stratification and constant meridional shear in the relative zonal velocity. It is easily seen that, with respect to the traditional hydrostatic equatorial $\beta$-plane equations, this configuration is inertially unstable in an interval between the equator and a latitude proportional to the value of the velocity shear. Unstable solutions to the linearized equations exist for all nonzero values of velocity shear, and the fastest growing modes for each vertical scale exhibit a column of vertically stacked cells of overturning motion in the verticalmeridional plane, known as Taylor vortices, centered over the unstable region-a pattern familiar from unstable Taylor-Couette flow between coaxial rotating cylinders. The corresponding pattern in the zonal velocity perturbation field is a column of alternately signed jets over the equatorward side of the unstable region and, in the potential temperature perturbation field, two columns of alternately signed anomalies, antisymmetric about the unstable region and $90^{\circ}$ out of phase with the zonal velocity anomaly (see Fig. 2).

Hitchman et al. (1987) identified the characteristic inertial instability pattern in Northern Hemisphere winter temperature data from satellite observations of the 

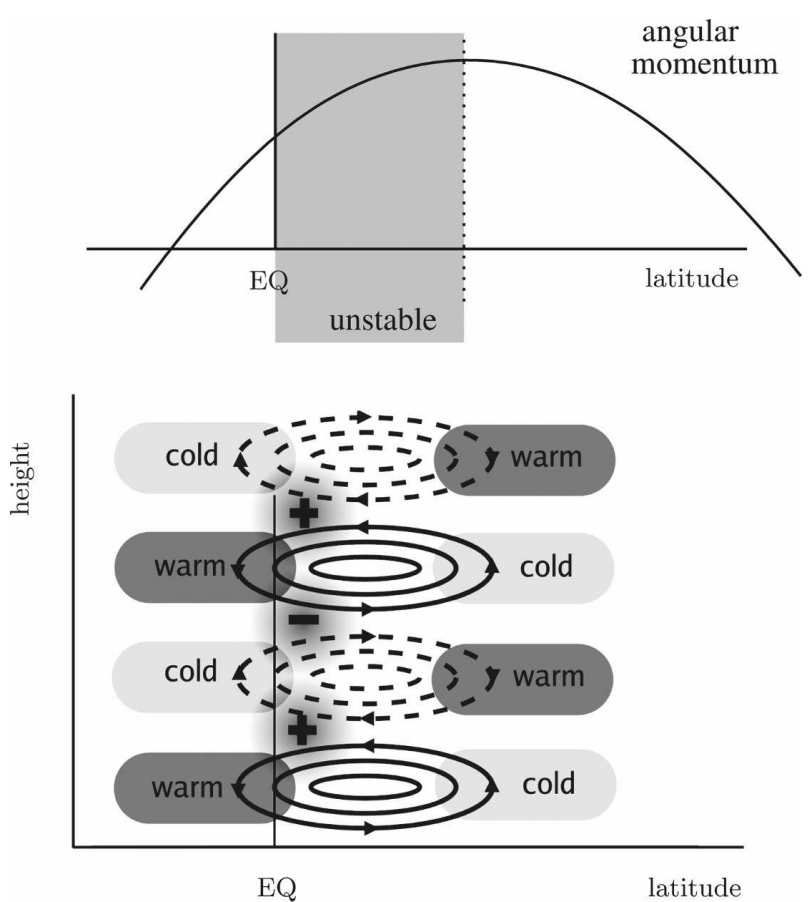

FIG. 2. Schematic diagram showing features of the Dunkerton (1981) solution to the primitive equations linearized about a basic state with linear meridional shear in the zonal velocity and stable stratification. The basic state is inertially unstable in the shaded interval. The fastest growing mode features a single column of Taylor vortices over the unstable region. Solid (dashed) contours are vortices with anticlockwise (clockwise) circulation. The shaded circles with + and - represent eastward and westward anomalies in the zonal wind on the equatorward side of the unstable region associated with, respectively, equatorward and poleward transport of angular momentum. The cold and warm cells represent the "pancake structures" in the temperature field associated with, respectively, upward and downward motion.

lower mesosphere. Oppositely signed columns of temperature anomalies with amplitudes of about $5 \mathrm{~K}$ and vertical wavelengths of about $15 \mathrm{~km}$ were observed over the equator, persisting for periods of one to two weeks. Similar features were identified by Hayashi et al. (1998), with further evidence that they represent inertial instability provided by corresponding out of phase patterns in winter midlatitudes, consistent with the Dunkerton solution. In both studies, the events were seen to be preceded by breaking Rossby waves at midlatitudes, which pull air with negative (positive) potential vorticity into the Northern (Southern) Hemisphere over a longitude interval of about $60^{\circ}$, thus violating the symmetric stability condition. The signature of inertial instability activity is routinely noted in numerical studies of the middle atmosphere (e.g., Hunt 1981).

In addition, the observed temperature and momentum fields in the middle atmosphere suggest that iner- tial adjustment is an important part of the general circulation during solstice seasons, when solar forcing is maximum in the summer hemisphere. In other words, the system is constantly being forced toward an inertially unstable radiative equilibrium state and adjusting itself toward a dynamically stable or neutral state featuring approximately flat meridional profiles of temperature and angular momentum throughout the equatorial region (Dunkerton 1989; Semeniuk and Shepherd 2001).

It should be noted that the observed fields suggesting inertial instability are themselves rarely globally axisymmetric. Indeed, the role of breaking Rossby waves in precipitating inertial instability events ensures that in nature they are quite asymmetric (Knox and Harvey 2005). While it can be argued that the relevant physics is largely contained in the meridional and vertical gradients of angular momentum and entropy and can be well represented by an axisymmetric treatment, studies (e.g., Dunkerton 1983, 1993) have shown that there are circumstances when asymmetric, propagating modes of instability dominate over symmetric instability modes.

In this paper, the problem of the stability conditions for a zonally symmetric equilibrium flow in a shallow atmosphere is revisited, but without invoking the traditional approximation or hydrostatic balance. An equatorial $\beta$ plane is used, which simplifies the mathematics compared to the corresponding problem in spherical geometry and adequately accounts for the effect of the nontraditional Coriolis terms. Exploiting the conservation properties of the inviscid, adiabatic, zonally symmetric compressible Euler equations, an adaptation of the energy-Casimir method (Holm et al. 1985; Shepherd 1990; Cho et al. 1993) is applied. The method entails finding a functional conserved by the dynamics and the class of equilibrium solutions at which the functional takes local extrema. It is then shown that such equilibria are stable with respect to small amplitude perturbations.

In section 2, the Euler equations on a generalized equatorial $\beta$ plane are presented along with their conservation laws. In section 3 conditions for linear stability are derived using the energy-Casimir method. Examples, including the Dunkerton case of linear zonal velocity shear, are presented in section 4 . Finally, in section 5 the difficulty of extending the result to apply to stability with respect to perturbations of finite amplitude is discussed.

\section{Symmetric Euler equations}

Consider adiabatic, compressible $x$-independent flow in a domain with rectangular cross section 
$\mathcal{D}=\{(y, z) \mid-L \leq y \leq L, 0 \leq z \leq H\}$ governed by the Euler equations,

$$
\begin{aligned}
u_{t} & =-v u_{y}-w u_{z}+\beta y v-\gamma w, \\
v_{t} & =-v v_{y}-w v_{z}-\beta y u-\frac{1}{\rho} p_{y}, \\
w_{t} & =-v w_{y}-w w_{z}+\gamma u-g-\frac{1}{\rho} p_{z}, \\
\rho_{t} & =-(\rho v)_{y}-(\rho w)_{z}, \quad \text { and } \\
\eta_{t} & =-v \eta_{y}-w \eta_{z},
\end{aligned}
$$

where $u, v$, and $w$ are the components of velocity in the $x, y$, and $z$ directions; $\rho, p$, and $\eta$ are density, pressure, and entropy; and $\beta \equiv 2 \Omega / a$ and $\gamma \equiv 2 \Omega$ are the parameters of the Coriolis force, with $\Omega$ the angular rotation rate of the planet and $a$ its radius. Subscripts denote partial differentiation. For convenience, we assume the no-normal-flow boundary condition $v=0$ on $y= \pm L$ and $w=0$ on $z=0, H$. This is not a restriction on possible instability because symmetric instability is a local (parcel) instability (Holt and Thorpe 1991; Cho et al. 1993). If the domain were instead chosen to be infinite in $y$, as is commonly the case for analyzing equatorially trapped linear wave solutions to (2.12), the appropriate boundary condition would be that the velocity vanish as $y \rightarrow \pm \infty$. The conserved integrals defined below would still be conserved under this boundary condition, and the stability conditions derived unchanged.

The system is closed by an equation of state

$$
F(\rho, p, \eta)=0,
$$

such as the ideal gas law.

The equations conserve energy

$\mathcal{H}[\mathbf{x}]=\iint_{\mathcal{D}} \rho\left[\frac{1}{2}\left(u^{2}+v^{2}+w^{2}\right)+g z+\mathcal{E}(\rho, \eta)\right] d y d z$,

where $\mathbf{x} \equiv(u, v, w, \rho, \eta)$ and $\mathcal{E}(\rho, \eta)$ is internal energy, satisfying the thermodynamic identity

$$
d \mathcal{E}=\frac{p}{\rho^{2}} d \rho+\tau d \eta
$$

where $\tau(\rho, \eta)$ is temperature. Also conserved are functionals of the form

$$
C[\mathbf{x}]=\iint_{\mathcal{D}} \rho C(m, \eta) d y d z,
$$

where $C(m, \eta)$ is any function, and $m$ is defined by

$$
m \equiv u-\frac{1}{2} \beta y^{2}+\gamma z
$$

Up to an additive constant, $m$ is proportional to the $\beta$-plane approximation to the component of absolute angular momentum parallel to the earth's rotation axis (Grimshaw 1975) and will be referred to hereafter simply as angular momentum. Note the term $\gamma z$ in (2.6), which is absent when the traditional approximation is made.

Functionals of the form (2.5) are related to the Casimir invariants of the noncanonical Hamiltonian representation of the system (2.1) (Shepherd 1990), and their conservation is a consequence of the material conservation of $m$ and $\eta$ by the flow. We note also that fluid parcels conserve potential vorticity

$$
q=\frac{1}{\rho} \partial(\eta, m)
$$

where $\partial(\cdot, \cdot)$ is the Jacobian operator, defined by

$$
\partial(f, g) \equiv f_{y} g_{z}-f_{z} g_{y}=(\nabla f \times \nabla g) \cdot \mathbf{i},
$$

where $\mathbf{i}$ is the unit vector in the $x$ direction. The sign of $\partial(f, g)$ is given by the "right-hand rule" applied to $\nabla f$ and $\nabla g$ : positive if $\nabla f$ points in the semicircle clockwise of $\nabla g$.

\section{a. Steady solution and linearized equations}

We seek to determine the stability of an equilibrium solution (the "basic state") $\mathbf{x}=\mathbf{X}$ of (2.1) with $u=U(y$, $z), v=w=0, \eta=N(y, z)$, and $\rho=D(y, z)$, with associated $m=M(U ; y, z)$ and $\tau=T(D, N)$. The pressure field $P(D, N)$ balances the velocity and mass fields: from (2.1b) and (2.1c),

$$
\begin{aligned}
& -\beta y U-\frac{1}{D} P_{y}=0, \quad \text { and } \\
& \gamma U-g-\frac{1}{D} P_{z}=0,
\end{aligned}
$$

which may be combined to obtain the thermal wind balance relation

$$
\partial\left[U, M^{(p)}\right]=\frac{1}{D^{2}} \partial(P, D),
$$

where

$$
M^{(p)} \equiv-\frac{1}{2} \beta y^{2}+\gamma z
$$

is the planetary angular momentum. Equation (2.10) relates the basic-state baroclinicity vector $\nabla P \times \nabla D$ to the basic-state velocity field. 


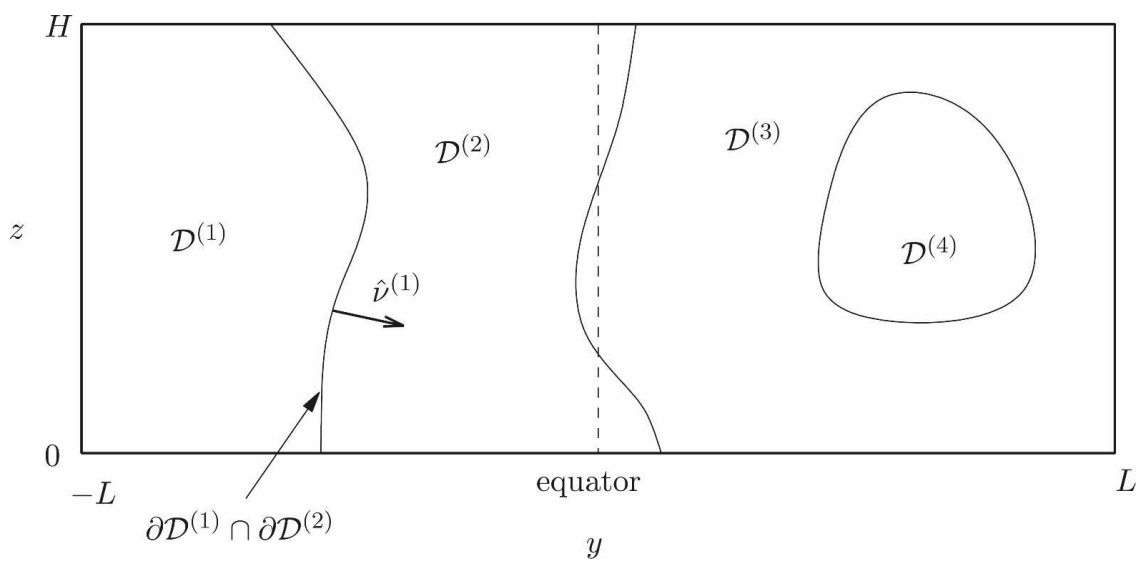

FIG. 3. Sample partition of $\mathcal{D}$ into regions with nonzero $Q$.

The system (2.1) linearized about $\mathbf{X}$ is

$$
\begin{aligned}
u_{t}^{\prime} & =-U_{y} v^{\prime}-U_{z} w^{\prime}+\beta y v^{\prime}-\gamma w^{\prime}, \\
v_{t}^{\prime} & =-\beta y u^{\prime}-\frac{1}{D} p_{y}^{\prime}+\frac{1}{D^{2}} P_{y} \rho^{\prime}, \\
w_{t}^{\prime} & =\gamma u^{\prime}-\frac{1}{D} p_{z}^{\prime}+\frac{1}{D^{2}} P_{z} \rho^{\prime}, \\
\rho_{t}^{\prime} & =-\left(D v^{\prime}\right)_{y}-\left(D w^{\prime}\right)_{z}, \quad \text { and } \\
\eta_{t}^{\prime} & =-N_{y} v^{\prime}-N_{z} w^{\prime},
\end{aligned}
$$

where $\mathbf{x}^{\prime} \equiv\left(u^{\prime}, v^{\prime}, w^{\prime}, \rho^{\prime}, \eta^{\prime}\right)$ represents the departure from $\mathbf{X}$, and the perturbation pressure $p^{\prime}$ is related to the perturbation density $\rho^{\prime}$ and entropy $\eta^{\prime}$ by

$$
p^{\prime}=\left(\frac{\partial P}{\partial D}\right)_{N} \rho^{\prime}+\left(\frac{\partial P}{\partial N}\right)_{D} \eta^{\prime},
$$

where $\left.(\partial P / \partial D)_{N} \equiv(\partial p / \partial \rho)_{\eta}\right|_{(D, N)}$, etc.

We assume that the mapping from $(y, z)$ to $(M, N)$ has nonzero Jacobian everywhere in $\mathcal{D}$ except perhaps on a finite number of curves and partition $\mathcal{D}$ accordingly into a finite number of subregions $\mathcal{D}^{(i)}(i=1, \ldots$, $n$ ), such that

$$
Q \equiv \frac{1}{D} \partial(N, M) \neq 0
$$

inside each of the $\mathcal{D}^{(i)}$ (see Fig. 3): $Q$ is the potential vorticity associated with the basic state. By construction, the mapping from $(y, z)$ to $(M, N)$ has a unique inverse inside each $\mathcal{D}^{(i)}$, which we denote by $\left[Y^{(i)}(M\right.$, $\left.N), Z^{(i)}(M, N)\right]$.

\section{b. A conservation law for the linearized dynamics}

We employ the energy-Casimir approach to derive linear stability conditions. This method normally in- volves constructing a functional that is exactly conserved by the nonlinear equations and has a critical point (in the sense of functionals) at the basic state (Holm et al. 1985; Cho et al. 1993). The functional typically takes the form of a sum of the Hamiltonian and a Casimir invariant, and its second variation evaluated at the basic state is an exact quadratic invariant of the corresponding linearized equations. Conditions for the second variation to be sign-definite correspond to the condition that the basic state be a local minimum or maximum of the conserved functional, and imply linear stability of the basic state.

In our case, finding such a functional is problematic (see section 5 below), but we can still apply this "nonlinear method" to the linear problem using a functional that is not conserved by the nonlinear equations, but whose second variation about the basic state is a quadratic invariant of the linearized equations. Consider the "Casimir"

$$
C_{L}=\sum_{i=1}^{n} \iint_{\mathcal{D}^{(i)}} \rho C^{(i)}(m, \eta) d y d z
$$

where each of the $C^{(i)}$ are arbitrary twice-differentiable functions of $m$ and $\eta$. We observe that unless $Q$ is nowhere zero, in which case there is only a single subdomain, $C_{L}$ is not conserved by the nonlinear system (2.1) and hence not really a Casimir invariant. Differentiating $C_{L}$ with respect to time and substituting from (2.1) gives

$$
\frac{d}{d t} C_{L}=\frac{1}{2} \sum_{i, j=1}^{n} \int_{\partial \mathcal{D}^{(i)} \cap \partial \mathcal{D}^{(j)}} \rho\left[C^{(j)}-C^{(i)}\right] \boldsymbol{v} \cdot \hat{\boldsymbol{v}}^{(i)} d l^{(i)}(y, z),
$$

where $\boldsymbol{v} \equiv(v, w), \partial \mathcal{D}^{(i)}$ is the boundary of the region $\mathcal{D}^{(i)}, \hat{v}^{(i)}$ is the outward-pointing unit vector normal to 
$\partial \mathcal{D}^{(i)}$, and $d l^{(i)}$ is the element of arclength along $\partial \mathcal{D}^{(i)}$. The outer boundary terms vanish because the velocity $\boldsymbol{v}$ is tangential to the boundary of $\mathcal{D}$ by assumption. The quantity $d C_{L} / d t$ vanishes if the functions $C^{(i)}$ in neighboring regions always match along the boundaries but, since the $C^{(i)}$ are in general different, this is not generally the case once the system is displaced from $\mathbf{X}$.

We nevertheless choose the functions $C^{(i)}$ so that $C_{L}$ is tangent to $\mathcal{H}$ (in the sense of functionals) at the basic state. This ensures that the combined functional $\mathcal{H}+C_{L}$ has a critical point at the basic state, a prerequisite for it having a minimum or maximum. That is,

$$
\left.\delta\left(\mathcal{H}+C_{L}\right)\right|_{\mathbf{x}}=0
$$

where $\delta\left(\mathcal{H}+\mathcal{C}_{L}\right)_{\mathbf{X}}$ is the first variation of $\mathcal{H}+\mathcal{C}_{L}$ evaluated at $\mathbf{X}$. For arbitrary variation $\delta \mathbf{x} \equiv(\delta u, \delta v, \delta w, \delta \rho$, $\delta \eta)$

$$
\begin{aligned}
\left.\delta\left(\mathcal{H}+C_{L}\right)\right|_{\mathbf{x}}= & \sum_{i=1}^{n} \iint_{\mathcal{D}^{(i)}}\left(\delta \rho\left[\frac{1}{2} U^{2}+g z+\mathcal{E}(D, N)+D \mathcal{E}_{\rho}(D, N)+C^{(i)}(M, N)\right]+D\left\{\left[U+C_{m}^{(i)}(M, N)\right] \delta u\right.\right. \\
& \left.\left.+\left[\mathcal{E}_{\eta}(D, N)+C_{\eta}^{(i)}(M, N)\right] \delta \eta\right\}\right) d y d z .
\end{aligned}
$$

Hence $\left.\delta\left(\mathcal{H}+C_{L}\right)\right|_{\mathbf{x}}$ vanishes if

$$
C^{(i)}(M, N)=-\left[\frac{1}{2} U^{2}+g z+\mathcal{E}(D, N)+D \mathcal{E}_{\rho}(D, N)\right]
$$

$C_{m}^{(i)}(M, N)=-U, \quad$ and

$C_{\eta}^{(i)}(M, N)=-\mathcal{E}_{\eta}(D, N)$ for each $i$. Note that $z, U(y, z)$, and $D(y, z)$ are implicit functions of $(M, N)$ in each $\mathcal{D}^{(i)}$ through the inverse mappings $[y, z]=\left[Y^{(i)}(M, N), Z^{(i)}(M, N)\right]$. It may be verified that the conditions (2.19) are mutually consistent by differentiating $C^{(i)}(M, N)$ and using (2.10).

We now construct the quadratic invariant of the linearized equations based on the second variation of $\mathcal{H}+$ $C_{L}$ evaluated at $\mathbf{X}$, which is

$$
\begin{aligned}
\left.\delta^{2}\left(\mathcal{H}+C_{L}\right)\right|_{\mathbf{X}}= & \sum_{i=1}^{n} \iint_{\mathcal{D}^{(i)}}\left\{D\left[1+C_{m m}^{(i)}(M, N)\right](\delta u)^{2}+D(\delta v)^{2}+D(\delta w)^{2}+\left[2 \mathcal{E}_{\rho}(D, N)+D \mathcal{E}_{\rho \rho}(D, N)\right](\delta \rho)^{2}\right. \\
& \left.+D\left[\mathcal{E}_{\eta \eta}(D, N)+C_{\eta \eta}^{(i)}(M, N)\right](\delta \eta)^{2}+2 D \mathcal{E}_{\rho \eta}(D, N) \delta \rho \delta \eta+2 D C_{m \eta}^{(i)}(M, N) \delta u \delta \eta\right\} d y d z .
\end{aligned}
$$

The second partial derivatives of $C^{(i)}(m, \eta)$ at $(M, N)$ are obtained by differentiating $(2.19 \mathrm{~b})$ and $(2.19 \mathrm{c})$, giving

$$
\begin{aligned}
& C_{m m}^{(i)}(M, N)=-1+\frac{1}{D Q}\left(\beta y N_{z}+\gamma N_{y}\right), \\
& C_{m \eta}^{(i)}(M, N)=\frac{1}{D Q}\left(-\beta y M_{z}-\gamma M_{y}\right), \\
& C_{\eta m}^{(i)}(M, N)=\frac{1}{D Q} \mathcal{E}_{\eta \rho}(D, N)\left(D_{y} N_{z}-D_{z} N_{y}\right), \text { and }
\end{aligned}
$$

$$
\begin{aligned}
C_{\eta \eta}^{(i)}(M, N)= & -\mathcal{E}_{\eta \eta}(D, N) \\
& -\mathcal{E}_{\eta \rho}(D, N) \frac{1}{D Q}\left(D_{y} M_{z}-D_{z} M_{y}\right)
\end{aligned}
$$

since $-D Q$ is the Jacobian of the transformation from $(y, z)$ to $(M, N)$.

Identifying the primed variables in the linear system (2.12) with the variation $\delta \mathbf{x}$ in (2.20), we define $\mathcal{H}_{L}\left[\mathbf{x}^{\prime}\right.$; $\mathbf{X}]\left.\equiv \delta^{2}\left(\mathcal{H}+C_{L}\right)\right|_{\mathbf{X}}[\delta \mathbf{x} ; \mathbf{X}]$. Using (2.21), the nonormal-flow boundary condition, and standard thermodynamics relations, it can be shown that $\mathcal{H}_{L}$ is conserved by (2.12). 


\section{Conditions for linear stability}

We rewrite $\mathcal{H}_{L}$ so that the coefficients of the perturbation quantities all have the same dimensions:

$$
\begin{aligned}
\mathcal{H}_{L}\left[\mathbf{x}^{\prime} ; \mathbf{X}\right]= & \sum_{i=1}^{n} \iint_{\mathcal{D}^{(i)}}\left\{D U_{0}^{2}\left[1+C_{m m}^{(i)}(M, N)\right]\left(\frac{u^{\prime}}{U_{0}}\right)^{2}+D v^{\prime 2}+D w^{\prime 2}+D_{0}^{2}\left[2 \mathcal{E}_{\rho}(D, N)+D \mathcal{E}_{\rho \rho}(D, N)\right]\left(\frac{\rho^{\prime}}{D_{0}}\right)^{2}\right. \\
& +D N_{0}^{2}\left[\mathcal{E}_{\eta \eta}(D, N)+C_{\eta \eta}^{(i)}(M, N)\right]\left(\frac{\eta^{\prime}}{N_{0}}\right)^{2}+2 D D_{0} N_{0} \mathcal{E}_{\rho \eta}(D, N) \frac{\rho^{\prime} \eta^{\prime}}{D_{0} N_{0}} \\
& \left.+2 D U_{0} N_{0} C_{m \eta}^{(i)}(M, N) \frac{u^{\prime} \eta^{\prime}}{U_{0} N_{0}}\right\} d y d z
\end{aligned}
$$

where $U_{0}, D_{0}$, and $N_{0}$ are arbitrary positive constants with the dimensions of velocity, density, and entropy, respectively. Here, $\mathcal{H}_{L}$ will be strictly positive if the integrands are strictly positive, or equivalently, if $\left(U_{0}, D_{0}, N_{0}\right)$ can be found such that the matrices

$$
\Lambda^{(i)}(D, N, M)=\left[\begin{array}{ccc}
\frac{D_{0}^{2}}{D^{2}} c_{s}^{2}(D, N) & D_{0} N_{0} \mathcal{E}_{\rho \eta}(D, N) & 0 \\
D_{0} N_{0} \mathcal{E}_{\rho \eta}(D, N) & N_{0}^{2}\left[\mathcal{E}_{\eta \eta}(D, N)+C_{\eta \eta}^{(i)}(M, N)\right] & U_{0} N_{0} C_{m \eta}^{(i)}(M, N) \\
0 & U_{0} N_{0} C_{m \eta}^{(i)}(M, N) & U_{0}^{2}\left[1+C_{m m}^{(i)}(M, N)\right]
\end{array}\right]
$$

where $c_{s}^{2} \equiv(\partial P / \partial D)_{N}$ is the square of the speed of sound, are positive definite for all triples $(D, N, M)$ that occur in the corresponding region $\mathcal{D}^{(i)}$.

The condition for all of the $\boldsymbol{\Lambda}^{(i)}$ to be positive definite is equivalent to the condition that all subdeterminants that include the top left (or bottom right) element be positive (see, e.g., Perlis 1952, p. 103). Hence,

$$
\begin{gathered}
\frac{D_{0}^{2}}{D^{2}} c_{s}^{2}(D, N)>0, \\
\operatorname{det}\left[\begin{array}{cc}
\frac{D_{0}^{2}}{D^{2}} c_{s}^{2}(D, N) & D_{0} N_{0} \mathcal{E}_{\rho \eta}(D, N) \\
D_{0} N_{0} \mathcal{E}_{\rho \eta}(D, N) & N_{0}^{2}\left[\mathcal{E}_{\eta \eta}(D, N)+C_{\eta \eta}^{(i)}(M, N)\right]
\end{array}\right] \\
>0, \quad \text { and } \\
\operatorname{det} \Lambda^{(i)}(D, M, N)>0 .
\end{gathered}
$$

We will see later that (3.3) can be rephrased in terms of the geometric properties of the basic state, notably the gradients of $M(y, z)$ and $N(y, z):(3.3 \mathrm{~b})$ is a statement of inertial stability and (3.3c) of the symmetric stability condition on potential vorticity. But before elaborating on the interpretation of the conditions, we show that positive definiteness of $\mathcal{H}_{L}$ implies the stability of $\mathbf{x}^{\prime}=0$ with respect to a suitably defined norm.

Under the hypothesis that the $\Lambda^{(i)}$ are symmetric, positive-definite matrices, their eigenvalues $\lambda^{(i)}$ are all positive, and there exists a complete set of mutually orthogonal eigenvectors $\boldsymbol{\xi}_{j}[j=1,2,3]$ for each $\boldsymbol{\Lambda}^{(i)}$. Any vector $\mathbf{x}_{1}^{\prime} \equiv\left(\rho^{\prime} / D_{0}, \eta^{\prime} / N_{0}, u^{\prime} / U_{0}\right)$ can be written as a linear combination of the $\xi_{j}$ (for any particular $i$ ). Thus

$$
\mathbf{x}_{1}^{\prime}=\sum_{j=1}^{3} \alpha_{j} \xi_{j}
$$

and

$$
\mathbf{x}_{1}^{\prime T} \Lambda^{(i)} \mathbf{x}_{1}^{\prime}=\sum_{j=1}^{3} \lambda_{j}^{(i)} \alpha_{j}^{2}\left|\xi_{j}\right|^{2}
$$

because the $\xi_{j}$ are mutually orthogonal, and

$$
\lambda_{\min }^{(i)}\left|\mathbf{x}_{1}^{\prime}\right|^{2} \leq \mathbf{x}_{1}^{\prime T} \Lambda^{(i)} \mathbf{x}_{1}^{\prime} \leq \lambda_{\max }^{(i)}\left|\mathbf{x}_{1}^{\prime}\right|^{2},
$$

where $\lambda_{\min }^{(i)}$ and $\lambda_{\max }^{(i)}$ are the smallest and largest of the eigenvalues of $\Lambda^{(i)}$ [that a largest eigenvalue exists requires that the elements of $\boldsymbol{\Lambda}^{(i)}$ are finite for all $(y, z)$ in 
the domain $\mathcal{D}^{(i)}$. Note that because the matrices vary with $D, N$, and $M$, so do the eigenvalues. Let $\lambda_{-}$and $\lambda_{+}$ be the smallest and largest eigenvalues of the $\Lambda^{(i)}$ considering all triples $(D, N, M)$ and all $i$. Defining the norm $\left\|\mathbf{x}^{\prime}\right\|_{\lambda}$ by

$$
\begin{aligned}
\left\|\mathbf{x}^{\prime}\right\|_{\lambda}^{2}= & \iint_{\mathcal{D}} D\left\{\lambda\left[\left(\frac{\rho^{\prime}}{D_{0}}\right)^{2}+\left(\frac{\eta^{\prime}}{N_{0}}\right)^{2}+\left(\frac{u^{\prime}}{U_{0}}\right)^{2}\right]\right. \\
& \left.+v^{\prime 2}+w^{\prime 2}\right\} d y d z
\end{aligned}
$$

we have

$$
\left\|\mathbf{x}^{\prime}\right\|_{\lambda_{-}}^{2} \leq \mathcal{H}_{L}\left(\mathbf{x}^{\prime}\right) \leq\left\|\mathbf{x}^{\prime}\right\|_{\lambda_{+}}^{2} .
$$

But this is true for all time, and, since $\mathcal{H}_{L}$ is conserved in time, we have

$$
\begin{aligned}
\left\|\mathbf{x}^{\prime}(t)\right\|_{\lambda_{-}}^{2} & \leq \mathcal{H}_{L}\left[\mathbf{x}^{\prime}(t)\right]=\mathcal{H}_{L}\left[\mathbf{x}^{\prime}(0)\right] \leq\left\|\mathbf{x}^{\prime}(0)\right\|_{\lambda_{+}}^{2} \\
& \leq \frac{\lambda_{+}}{\lambda_{-}}\left\|\mathbf{x}^{\prime}(0)\right\|_{\lambda_{-}}^{2}
\end{aligned}
$$

so that for any $\varepsilon$, if $\left\|\mathbf{x}^{\prime}(0)\right\|_{\lambda_{-}}<\left(\lambda_{-} / \lambda_{+}\right)^{1 / 2} \varepsilon$, then $\left\|\mathbf{x}^{\prime}(t)\right\|_{\lambda_{-}}<\varepsilon$ for all $t$.

Therefore, if the conditions (3.3) are satisfied and all coefficients of disturbance quantities in (3.1) are bounded, then $\mathbf{x}^{\prime}=0$ is stable, and we may say that the solution to the full equations of disturbance quantities in (3.1) $\mathbf{x}=\mathbf{X}$ is linearly stable.

We now turn to the physical interpretation of the stability conditions (3.3). Equation (3.3a) is satisfied by any fluid. For example, for an ideal gas with equation of state

$$
p=R \rho \tau,
$$

where $R$ is a constant, it is readily shown that $c_{s}^{2}=$ $\left(c_{p} / c_{v}\right) R \tau>0$, where $c_{p}$ and $c_{v}$ are the specific heat capacities of the gas at constant pressure and volume, respectively.

Using (2.4), (2.21d), and (2.9), condition (3.3b) can be written

$$
\frac{1}{D^{3} Q}\left(\frac{\partial P}{\partial N}\right)_{D} \partial(M, P)>0,
$$

and condition $(3.3 \mathrm{c})$ can be written

$$
\frac{g \beta}{D}\left(\frac{\partial P}{\partial N}\right)_{D} \frac{y}{Q}>0 .
$$

Using thermodynamics identities, we find

$$
\left(\frac{\partial P}{\partial N}\right)_{D}=\left(\frac{\partial T}{\partial N}\right)_{D}\left(\frac{\partial P}{\partial T}\right)_{D}=\frac{T}{c_{v}}\left(\frac{\partial P}{\partial T}\right)_{D},
$$

where the heat capacity $c_{v}$ need not be constant, but is surely positive. The derivative $(\partial P / \partial T)_{D}$ is also posi-

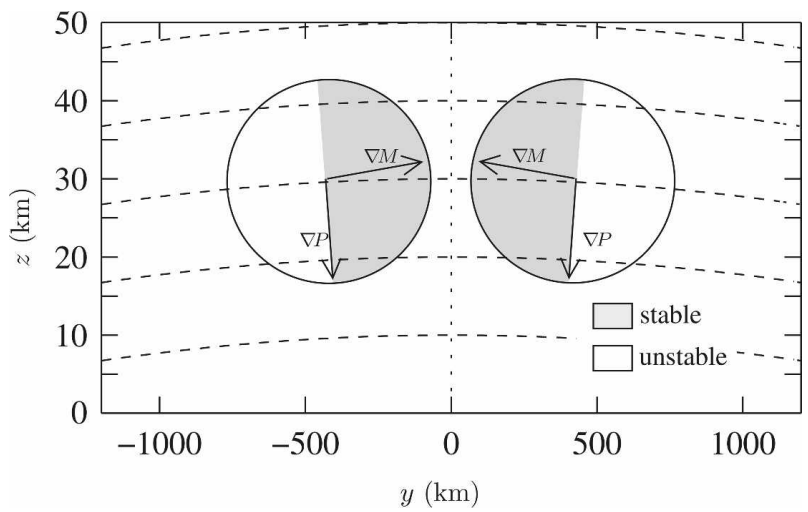

FIG. 4. Inertial stability condition. Dashed curves are contours of constant pressure with the curvature exaggerated by a factor of 10 compared to a typical atmospheric state. Pressure contours are concave down, implying $U>0$. For stability, the direction of $\nabla M$ must be in the shaded regions determined by $\nabla P$.

tive-pressure increases with increasing temperature for fixed density. Hence, $(\partial P / \partial N)_{D}>0$.

Equation (3.12) is therefore the well-known symmetric stability condition that potential vorticity be positive (negative) in the Northern (Southern) Hemisphere (Stevens 1983). Assuming that $Q$ is continuous across the equator, this implies that $Q=0$ at the equator. Equation (3.11) may then be interpreted as a generalization of the Rayleigh criterion for inertial stability: the angular momentum gradient must be clockwise (anticlockwise) of the pressure gradient in the Northern (Southern) Hemisphere, when viewed with the Northern Hemisphere on the right (see Fig. 4). For example, if the pressure gradient is directly downward, then $\nabla M$ must be toward the equator for stability. The shape of the pressure contours depends on the velocity field $U$. From (2.9),

$$
\frac{P_{z}}{P_{y}}=\frac{g-\gamma U}{\beta y U}
$$

so the pressure contours are concave down for eastward flow $(U>0)$ and concave up for westward flow $(U<$ $0)$. The effect of $\gamma$ is to steepen the pressure contours for eastward flow and flatten them for westward flow (we have assumed that $g>\gamma|U|$ ). Since $\mathrm{g} / \gamma \approx 7 \times 10^{4}$ $\mathrm{m} \mathrm{s}^{-1}$, much greater than any plausible $U$, condition (3.11) is hardly changed from the traditional case.

Equations (3.11) and (3.12) are formally the same stability conditions as those of Bowman and Shepherd (1995), where the hydrostatic approximation is used, namely $\left.y(\partial M / \partial y)\right|_{p}<0$ and $y Q>0$, but, of course, the definitions of $M$ and $Q$ are different because of the difference in the geometry and in the assumptions underlying the dynamical equations. 


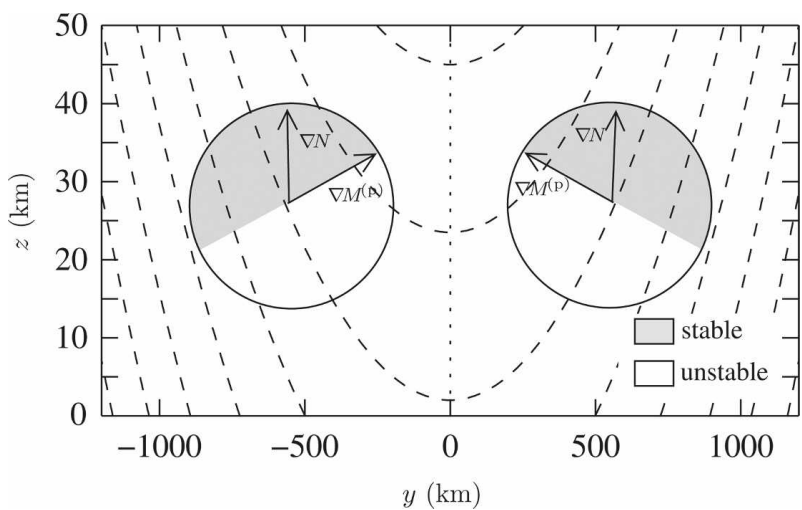

FIG. 5. Static stability condition. Dashed curves are contours of constant planetary angular momentum. For stability, the direction of $\nabla N$ must be in the shaded regions determined by $\nabla M^{(p)}$.

If the $\Lambda^{(i)}$ are positive definite, then it also follows from the theorem cited earlier that the bottom right element of $\boldsymbol{\Lambda}^{(i)}$ is positive; that is,

$$
1+C_{m m}^{(i)}(M, N)>0
$$

which, by (2.21a), is equivalent to

$$
\frac{1}{D Q}\left(\beta y N_{z}+\gamma N_{y}\right)=\frac{1}{D Q} \partial\left[N, M^{(p)}\right]>0,
$$

and may be interpreted as the condition that the component of the entropy gradient in the direction of the local planetary rotation vector

$$
\mathbf{\Omega}=\frac{1}{2}(\gamma \mathbf{j}+\beta y \mathbf{k})
$$

be positive (negative) in the Northern (Southern) Hemisphere. Here, $\mathbf{j}$ and $\mathbf{k}$ are the unit vectors in the $y$ and $z$ directions, respectively. Equivalently, the entropy gradient must be clockwise of the planetary angular momentum gradient in the Northern Hemisphere, and anticlockwise in the Southern Hemisphere (see Fig. 5). This is a generalization of static stability since the "static" state in the rotating frame is determined by the planetary rotation parameters $\beta$ and $\gamma$. For example, if the $\gamma$ term is neglected (as in the traditional hydrostatic primitive equations), then $\nabla M^{(p)}$ is toward the equator, and (3.16) reduces to $N_{z}>0$. Recall from our earlier discussion that an effect of $\gamma$ is to create the slope in the planetary angular momentum contours (see Fig. 1). The corresponding figure for the traditional case would have $\boldsymbol{\nabla} M^{(p)}$ horizontal and stability requiring that $\nabla N$ point in the upper half-circle. This effect of including the $\gamma$ terms was discussed by Sun (1995) in the context of an analysis of symmetric stability in the Boussinesq equations on an $f$ plane with the $\gamma$ terms. He found that

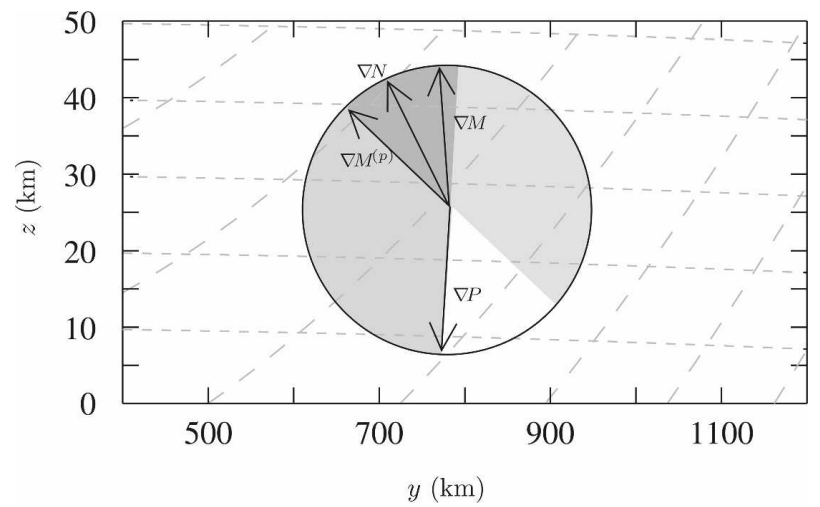

FIG. 6. An example of a basic state that satisfies the inertial and static stability conditions but fails the symmetric stability (potential vorticity) condition. The contours and shaded regions are as in Figs. 4 and 5. If they both fall in the darkly shaded region, it is possible to have both $\nabla N$ and $\nabla M$ satisfy the conditions for static and inertial stability, respectively, but be oriented relative to each other such that $Q$ has the wrong sign for stability.

the value of $N_{y}$, and in particular its sign, affects stability and the growth rate of instability if $\gamma$ is included.

Note that the potential vorticity condition (3.12) and either of (3.11) and (3.16) imply the remaining condition. However, it is possible for a state to be inertially and statically stable but to violate the potential vorticity condition. Such a situation is depicted in Fig. 6: both $\boldsymbol{\nabla} M$ and $\boldsymbol{\nabla} N$ point within their respective "semicircles of stability," but they are unstably oriented with respect to one another. Also recall that the coefficients in $\mathcal{H}_{L}$ have to be bounded to ensure that the matrices $\Lambda^{(i)}$ have a maximum eigenvalue. This potentially restricts the functional form of $M(y, z)$ and $N(y, z)$ in the limit as $y \rightarrow 0$.

Equivalent conditions to those derived here can be found using a heuristic approach based on forces on displaced fluid parcels, along the same lines as the method of Shutts and Cullen (1987). Considering an infinitesimal displacement from equilibrium of an infinitesimally thin line of fluid (a line and not a parcel because of the symmetry in $x$ ) such that its density and zonal velocity adjust in order to conserve entropy and angular momentum and assuming that the pressure field is unchanged by the perturbation, it can be shown that (3.11) and (3.12) imply that the component of net force on the displaced parcel in the direction of the displacement will be in the opposite sense to the displacement (Fruman 2005; §3.1.5). The advantage of the approach chosen in the present paper is that it is rigorous in the sense of not requiring any assumptions on the perturbations other than those implicit in the equations 
of motion. Furthermore, when applied to constrained models like the anelastic and Boussinesq equations, it can be extended to finite amplitude perturbations in certain circumstances. On the other hand, the parcel displacement approach has the advantage of predicting instability when the stability conditions are not satisfied, thus yielding necessary and sufficient conditions for stability when the perturbation does not alter the pressure field.

\section{Examples}

\section{a. Example 1: Isothermal ideal gas atmosphere in solid-body rotation}

We now show that an isothermal ideal gas atmosphere in solid-body rotation is linearly stable. In the equatorial $\beta$-plane system, the state corresponding to solid-body rotation is that with uniform zonal relative velocity $U=U_{00}$.

By the ideal gas equation of state (3.10) and the conditions for balance (2.9), we have

$$
\begin{aligned}
& \frac{\partial}{\partial y} \ln P=-\left(\frac{\beta U_{00}}{R T_{00}}\right) y, \quad \text { and } \\
& \frac{\partial}{\partial z} \ln P=-\left(\frac{g-\gamma U_{00}}{R T_{00}}\right),
\end{aligned}
$$

where $T_{00}$ is the uniform temperature from which we can solve for the basic-state pressure field:

$$
P(y, z)=P_{00} \exp \left[-\frac{1}{2}\left(\frac{\beta U_{00}}{R T_{00}}\right) y^{2}-\left(\frac{g-\gamma U_{00}}{R T_{00}}\right) z\right],
$$

where $P_{00}$ is the pressure at the origin. The potential vorticity satisfies

$$
D Q=\partial(N, M)=-\frac{1}{T_{00} D} \partial\left(P, M^{(p)}\right)=\left(\frac{\beta g}{T_{00}}\right) y,
$$

where $D=P / R T_{00}$.

The coefficient matrix $\Lambda$ for this state is [noting that since the basic state is an even function of $y$, there is only one function $C(m, \eta)$ required]

$$
\boldsymbol{\Lambda}=\left[\begin{array}{ccc}
D_{0}^{2} \frac{c_{p}}{c_{v}} \frac{R T_{00}}{D^{2}} & D_{0} N_{0} \frac{1}{c_{v}} \frac{R T_{00}}{D} & 0 \\
D_{0} N_{0} \frac{1}{c_{v}} \frac{R T_{00}}{D} & N_{0}^{2} \frac{T_{00}}{c_{v}} & 0 \\
0 & 0 & U_{0}^{2}
\end{array}\right],
$$

where we have used thermodynamics identities and (2.21). Choosing

$$
\begin{gathered}
N_{0}=\left(R c_{v}\right)^{1 / 2}, \quad U_{0}=\left(R T_{00}\right)^{1 / 2}, \quad \text { and } \\
D_{0}=\left(\frac{c_{v}}{R}\right)^{1 / 2} D_{00},
\end{gathered}
$$

where $D_{00} \equiv P_{00} / R T_{00}$, recalling that the constants $D_{0}$, $N_{0}$, and $U_{0}$ are arbitrary and are included to make the dimensions of the elements in $\Lambda$ uniform, we get

$$
\boldsymbol{\Lambda}=R T_{00}\left[\begin{array}{ccc}
\frac{c_{p}}{R}\left(\frac{D_{00}}{D}\right)^{2} & \frac{D_{00}}{D} & 0 \\
\frac{D_{00}}{D} & 1 & 0 \\
0 & 0 & 1
\end{array}\right]
$$

The eigenvalues of $\Lambda$ are $\lambda_{3}=R T_{00}$ and

$$
\begin{aligned}
\lambda_{2}= & \frac{1}{2}\left\{\left[\frac{c_{p}}{R}\left(\frac{D_{00}}{D}\right)^{2}+1\right]\right. \\
& \left. \pm \sqrt{\left[\frac{c_{p}}{R}\left(\frac{D_{00}}{D}\right)^{2}-1\right]^{2}+4\left(\frac{D_{00}}{D}\right)^{2}}\right\} R T_{00},
\end{aligned}
$$

which are positive for all $D>0$. The minimum value of $\lambda_{2}$ in the domain occurs at the origin and is equal to $\lambda_{3}$, and an upper bound on $\lambda_{1}$ is the trace of the upper-left principal subdeterminant of $\boldsymbol{\Lambda}$. We have, therefore, that the minimum and maximum eigenvalues are bounded from below and above by, respectively,

$$
\begin{aligned}
\lambda_{-}= & R T_{00}, \quad \text { and } \\
\lambda_{+}= & R T_{00}\left\{1+\frac{c_{p}}{R} \exp \left[\left(\frac{\beta U_{00}}{R T_{00}}\right) L^{2}\right.\right. \\
& \left.\left.+2\left(\frac{g-\gamma U_{00}}{R T_{00}}\right) H\right]\right\} .
\end{aligned}
$$

This confirms that the basic state is linearly stable.

\section{b. Example 2: Linear velocity shear at the equator and nearly uniform temperature}

We show that constant meridional velocity shear at the equator is inertially unstable. This is the case considered by Dunkerton (1981).

Consider again an ideal gas in equilibrium with temperature independent of height and velocity $U(y, z)=$ $\lambda y$, where $\lambda<g / \gamma L$ is a constant $[\lambda>g / \gamma L$ would imply negative temperatures in the domain; for earth values of $g$ and $\gamma$, and $L \approx 10^{3} \mathrm{~km}, g / \gamma L \sim 100\left(\mathrm{~m} \mathrm{~s}^{-1}\right) \mathrm{km}^{-1}$ ].

To proceed, we must specify pressure and density fields that are in balance with $U$ [i.e., which satisfy 
(2.9)]. Eliminating density $D$ between (2.9a) and (2.9b), we find

$$
\left(1-\frac{\gamma \lambda}{g} y\right) P_{y}+\left(-\frac{\beta \lambda}{g} y^{2}\right) P_{z}=0 .
$$

We solve for $P(y, z)$ using, for example, the method of characteristics (see, e.g., Zauderer 1989), finding

$$
\begin{aligned}
P(y, z)= & P_{00}\left(1-\frac{\gamma \lambda}{g} y\right)^{b} \\
& \times \exp \left\{-\frac{g}{R T_{00}}\left[z-\frac{\beta}{\gamma}\left(\frac{g}{\gamma \lambda} y+\frac{1}{2} y^{2}\right)\right]\right\},
\end{aligned}
$$

where

$$
b \equiv\left(\frac{g}{R T_{00}}\right)\left(\frac{\beta}{\gamma}\right)\left(\frac{g}{\gamma \lambda}\right)^{2} .
$$

The corresponding density and temperature fields are

$$
\begin{aligned}
& D(y, z)=\frac{P}{R T_{00}} \frac{1}{[1-(\gamma \lambda / g) y]}, \quad \text { and } \\
& T(y, z)=T_{00}\left(1-\frac{\gamma \lambda}{g} y\right) .
\end{aligned}
$$

Notice that there is a very slight meridional temperature gradient to balance the velocity shear (a physically plausible value of $\lambda$ over a substantial latitude range might be $\leqslant 10^{-4} \mathrm{~s}^{-1}$, making $\gamma \lambda / g \lesssim 10^{-9}$, a temperature gradient of $0.001 T_{00}$ per $1000 \mathrm{~km}$ ).

In Dunkerton (1981), it is shown that this basic-state velocity field is linearly unstable in a hydrostatic system, with perturbations leading to the formation of Taylor vortices in the latitude interval $0<y<\lambda / \beta$ and corresponding changes to the $m$ and $\eta$ fields. In the present system, the potential vorticity is

$$
Q=\frac{\beta g}{D T}\left[\left(1+\frac{\gamma \lambda^{2}}{\beta g}\right) y-\frac{\lambda}{\beta}\left(1+\frac{\gamma^{2}}{g^{2}} c_{p} T_{00}\right)\right] .
$$

The latitude $y_{0}$ at which $Q=0$ (the width of the unstable latitude interval) is

$$
y_{0}=\frac{\lambda}{\beta}\left(\frac{1+\frac{\gamma^{2}}{g^{2}} c_{p} T_{00}}{1+\frac{\gamma \lambda^{2}}{\beta g}}\right),
$$

which has its maximum value $(\approx \lambda / 2 \beta)$ for $\lambda=(g / a)^{1 / 2}$. For $\lambda \ll(g / a)^{1 / 2}, y_{0} \approx \lambda / \beta$, its value in the traditional system, and for $\lambda \gg(g / a)^{1 / 2}, y_{0} \rightarrow 0$ like $\lambda^{-1}$. For the $\gamma$ correction to the interval of anomalous potential vorticity to be significant, one would need $\lambda \sim(g / a)^{1 / 2}$. For earth, this is approximately $1\left(\mathrm{~m} \mathrm{~s}^{-1}\right) \mathrm{km}^{-1}$, which is possible, although not over a wide latitude interval as it would entail unrealistically large velocities. Hence, the result of Dunkerton for the traditional case is approximately valid for a large range of values of the horizontal shear.

The change in the width of the unstable latitude interval is due to the increasing curvature of the pressure contours with increasing velocity. At the equator, $\nabla P$ points vertically down, so the clockwise edge of the semicircle of stability (see Fig. 4) is vertically up; $\nabla M$ points clockwise of vertically up at the equator (for $\lambda>$ 0 ) and turns toward the vertical linearly with $y$. Meanwhile, the semicircle of stability rotates clockwise (toward $\nabla M)$ quadratically with $y$. Here $\nabla M$ enters the semicircle of stability at $y_{0}$.

\section{c. More examples}

The preceding two examples and three others are summarized in Table 1.

In example 3 , we consider an angular momentum field that decreases with latitude like $-\alpha|y|^{k}(\alpha>0, k$ $>1$ ). Such a solution is continuous and differentiable for all $y$ and satisfies the Rayleigh criterion for stability. For $1<k<2$, the solution is stable in the neighborhood of the equator for all $\alpha$. However, for $k=2$ and small enough $\alpha$, and for $k>2$ and all $\alpha$, there is an interval of anomalous potential vorticity straddling the equator. In particular, this implies that a flat angular momentum profile is not stable. This instability is neither inertial nor convective, since both (3.11) and (3.16) hold, but of the "symmetric" type illustrated in Fig. 6. This can be seen by looking at the gradients of $M$ and $N$ to leading order in $y$, normalized to have unit $z$ component, for $k>2$ :

$$
\begin{aligned}
& \boldsymbol{\nabla}_{n} M \sim-\left(\frac{\alpha k}{\gamma} \frac{|y|^{k}}{y}\right) \mathbf{j}+\mathbf{k}, \quad \text { and } \\
& \boldsymbol{\nabla}_{n} N \sim-\left(\frac{\beta \gamma c_{p} T_{00}}{g^{2}} y\right) \mathbf{j}+\mathbf{k},
\end{aligned}
$$

where $\boldsymbol{\nabla}_{n}$ is the normalized gradient. At the equator, both gradients are pointing upward. For small $y, \nabla_{n} N$ tips toward the equator linearly with $y$, faster than $\boldsymbol{\nabla}_{n} M$, implying anomalous $Q$ in both hemispheres. As an example, for the case $k=4$ and $\alpha=10^{-23}$, corresponding to an angular momentum difference between the equator and $y=1000 \mathrm{~km}$ of $10 \mathrm{~m} \mathrm{~s}^{-1}$ (a velocity difference of about $5 \mathrm{~m} \mathrm{~s}^{-1}$ ), the interval of instability is 
TABLE 1. Steady states of symmetric Euler system and corresponding stability criteria for 1) solid body rotation of isothermal atmosphere; 2) linear variation of velocity with latitude; 3) symmetric about the equator power-law variation of velocity with latitude; 4) linear temperature increase with height; and 5) linear variation of velocity and temperature with height.

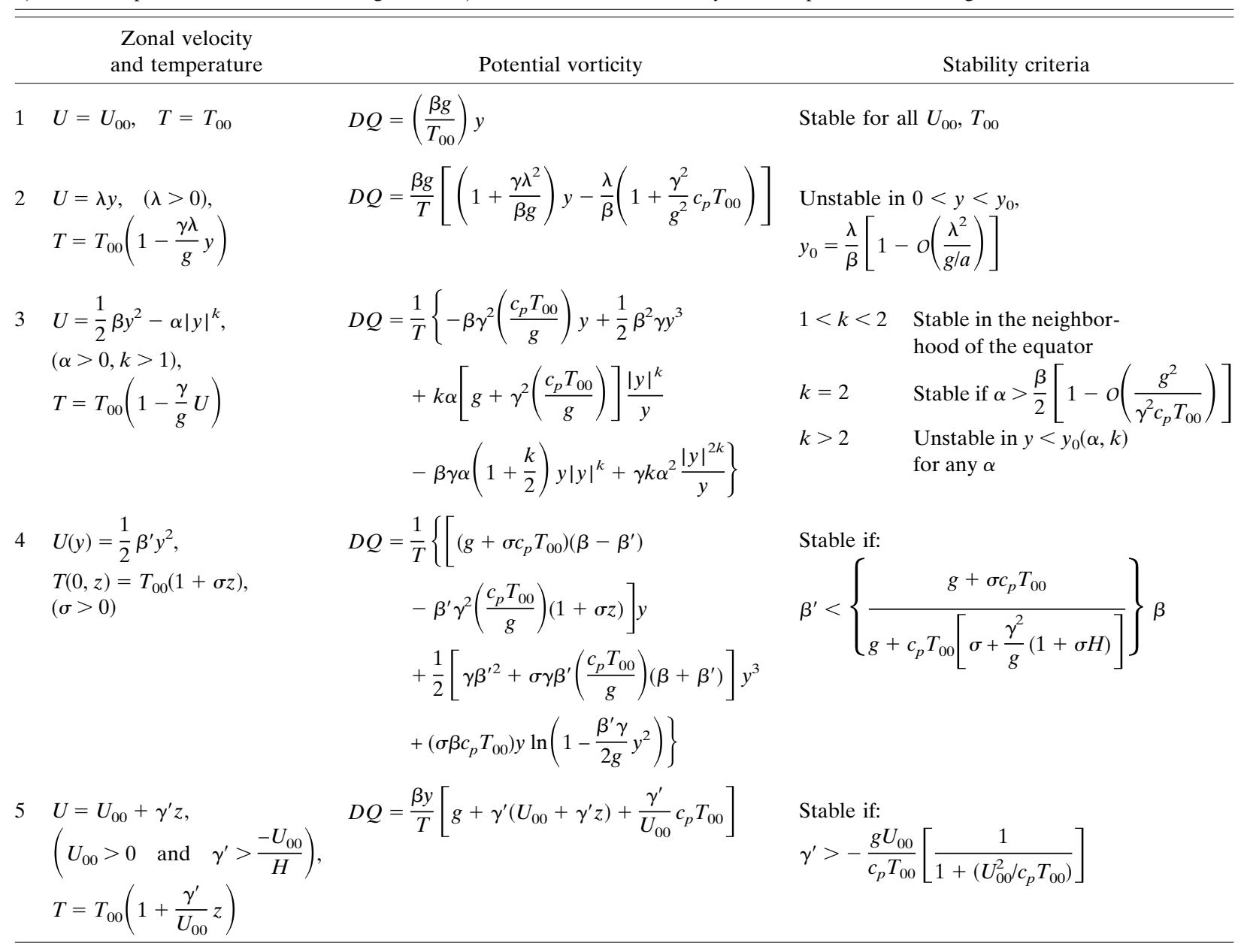

only about $100 \mathrm{~m}$ in width for typical earth values. This effect is dependent on the nontraditional terms-note the appearance of $\gamma$ in (4.15b). However, since inertial adjustment tends to be localized to the region of anomalous potential vorticity (Dunkerton 1981; Ooyama 1966), this instability is perhaps of little importance.

In example 4 , we consider a case with temperature varying with height and velocity independent of height. Temperature increasing with height is evidently slightly destabilizing near the equator, making the threshold angular momentum curvature $\beta-\beta^{\prime}$ slightly larger.

In example 5, we find that velocity and temperature decreasing with height is destabilizing, as we would expect because it entails angular momentum increasing more slowly with distance from the axis of rotation. Interestingly though, for the example considered, the stability criterion is independent of $\gamma$, the planetary angular momentum gradient with height.

\section{Remarks on nonlinear stability}

In similar problems (e.g., Cho et al. 1993; see also $\mathrm{Mu}$ et al. 1996), the conditions for linear stability can be extended to the problem of finite amplitude, "nonlinear" stability, meaning that if a basic state $\mathbf{X}$ satisfies appropriate conditions, then arbitrary, even large, perturbations to $\mathbf{X}$ will remain bounded for all time, as governed by the full nonlinear equations. The usual approach is to define an exact invariant $\mathcal{A}[\mathbf{x} ; \mathbf{X}]$, called the pseudoenergy, that vanishes if $\mathbf{x}=\mathbf{X}$ and to find conditions on $\mathbf{X}$ such that $\mathcal{A}$ is strictly positive for all other choices of $\mathbf{x}$ (Shepherd 1990).

Since linear stability is a necessary condition for nonlinear stability (provided the norm for the linear problem is the small amplitude limit of the finite amplitude norm in terms of which nonlinear stability is defined), in this case we only consider $\mathbf{X}$ having $Q=0$ on $y=0$ 
and nowhere else. Recalling that $q$ is materially conserved, one can show that the functional

$$
\begin{aligned}
C_{N L}= & \iint_{\mathcal{D}} \rho\left\{C^{-}(m, \eta)+H(q)\left[C^{+}(m, \eta)\right.\right. \\
& \left.\left.-C^{-}(m, \eta)\right]\right\} d y d z
\end{aligned}
$$

where

$$
H(q)= \begin{cases}0, & q<0 \\ 1, & q \geq 0,\end{cases}
$$

is conserved by (2.1). We then choose $C^{-}$and $C^{+}$to satisfy (2.19) and define

$$
\mathcal{A}[\mathbf{x} ; \mathbf{X}]=\left(\mathcal{H}+C_{N L}\right)[\mathbf{x}]-\left(\mathcal{H}+C_{N L}\right)[\mathbf{X}] .
$$

By construction, $\mathcal{A}[\mathbf{X} ; \mathbf{X}]=0$ and $\mathcal{A}$ has a critical point at $\mathbf{x}=\mathbf{X}$. However, it is not a simple matter to find norms to bound $\mathcal{A}$ from above and below for all times like we did with $\mathcal{H}_{L}$ in the linear case. There are two separate difficulties. The first is connected to the asymmetry of the basic state and hence to the difference between $C^{-}$and $C^{+}$. If the flow evolves in such a way that regions of $q>0$ develop in the Southern Hemisphere, then the corresponding contribution to $\mathcal{A}$ from those regions will depend on $C^{+}$and not on $C^{-}$as it did at the basic state. In this way, it is possible for $\mathcal{A}$ to be negative, which would prevent any rigorous Lyapunov stability result by this method. Note that this difficulty does not arise for a basic state for which $Q$ is nowhere zero, but such a case is not especially relevant to equatorial dynamics.

The second difficulty is related to the fact that we are free to choose $C^{-}$and $C^{+}$provided we satisfy (2.19) and the matching condition at the equator at equilibrium, but we cannot choose the dependence of the internal energy $\mathcal{E}$ on $\rho$ and $\eta$ and, hence, cannot bound certain terms in $\mathcal{A}$ for all possible perturbations of $\rho$ and $\eta$ outside of the ranges of $N(y, z)$ and $D(y, z)$. Thus, conditions for the positive definiteness of $\mathcal{A}$ depend not only on the basic state, but on the states that the system might pass through over time- the details of which we do not know. Note that this difficulty is avoided if either the Boussinesq or the anelastic approximation is used, in which case density is not a dependent variable.

Both difficulties are addressed in more detail and the corresponding problem for the anelastic equations explored in Fruman (2005).

\section{Summary}

Conditions for linear stability of a zonal flow equilibrium solution to the $x$-symmetric adiabatic compress- ible Euler equations on an equatorial $\beta$ plane, including a leading order representation of the nontraditional Coriolis force terms (controlled by the parameter $\gamma \equiv$ $2 \Omega$ ), have been calculated. The inertial stability condition from the traditional hydrostatic result is shown to extend, in a formal sense, to this system. The condition for stability is still the Rayleigh criterion that the angular momentum in the basic state increase toward the equator on surfaces of constant pressure, although the definition of angular momentum is slightly different with the inclusion of the nontraditional terms. The "static" stability condition derived is rather different from that in the traditional system due to the modification of the surfaces of constant planetary angular momentum due to the nontraditional terms. We find that, for static stability, the gradient of entropy must be clockwise of the gradient of planetary angular momentum in the Northern Hemisphere and anticlockwise in the Southern Hemisphere (viewed with the Northern Hemisphere on the right). The symmetric stability condition, that potential vorticity be positive (negative) in the Northern (Southern) Hemisphere, generalizes to the nontraditional system if the definition of potential vorticity is modified appropriately.

Several examples were presented. In each case, zonal velocity and temperature fields that satisfy thermal wind balance are chosen, and the potential vorticity is compared to the symmetric stability condition. The effect of the nontraditional terms is of particular interest in two of the examples. In the case of a basic state with angular momentum profile higher than quadratic in $y$, conditions for both inertial and static stability are satisfied, but the potential vorticity condition fails. We showed that this is due to the tipping of the angular momentum gradient more slowly than the entropy gradient as $y$ increases away from the equator, an effect dependent on the inclusion of the nontraditional terms in the dynamical equations. In the case of a basic state with linear velocity shear across the equator, the celebrated example of Dunkerton (1981), the dependence of the width of the latitude interval of instability on the velocity shear changes if $\gamma$ is included in the equations, approaching zero as the shear gets large.

Steps for extending the result to finite amplitude disturbances were outlined. However, technical details associated with asymmetric basic states and the evolution of the density field prevent a general nonlinear result.

Acknowledgments. This work was part of the Ph.D. dissertation of MDF and was supported by an Ontario Graduate Scholarship and grants from the Natural Sciences and Engineering Research Council of Canada and the Walter C. Sumner Foundation. 


\section{REFERENCES}

Bowman, J. C., and T. G. Shepherd, 1995: Nonlinear symmetric stability of planetary atmospheres. J. Fluid Mech., 296, 391407.

Cho, H.-R., T. G. Shepherd, and V. A. Vladimirov, 1993: Application of the direct Liapunov method to the problem of symmetric stability in the atmosphere. J. Atmos. Sci., 50, 822-836.

Colin de Verdière, A., and R. Schopp, 1994: Flows in a rotating spherical shell: The equatorial case. J. Fluid Mech., 276, 233260.

Dunkerton, T. J., 1981: On the inertial stability of the equatorial middle atmosphere. J. Atmos. Sci., 38, 2354-2364.

- 1983: A nonsymmetric equatorial inertial instability. $J$. Atmos. Sci., 40, 807-813.

__, 1989: Nonlinear Hadley circulation driven by asymmetric differential heating. J. Atmos. Sci., 46, 956-974.

_- 1993: Inertial instability of nonparallel flow on an equatorial $\beta$ plane. J. Atmos. Sci., 50, 2744-2758.

Durran, D. R., and C. Bretherton, 2004: Comments on "The roles of the horizontal component of the Earth's angular velocity in nonhydrostatic linear models." J. Atmos. Sci., 61, 19821986.

Eckart, C., 1960: Hydrodynamics of Oceans and Atmospheres. Pergamon Press, 290 pp.

Fruman, M. D., 2005: Equatorial symmetric stability. Ph.D. thesis, University of Toronto, $178 \mathrm{pp}$.

Gerkema, T., and V. I. Shrira, 2005: Near-inertial waves on the "nontraditional" $\beta$ plane. J. Geophys. Res., 110, C01003, doi:10.1029/2004JC002519.

Gill, A. E., 1982: Atmosphere-Ocean Dynamics. Academic Press, $662 \mathrm{pp}$.

Grimshaw, R. H. J., 1975: A note on the $\beta$-plane approximation. Tellus, 27, 352-357.

Hayashi, H., M. Shiotani, and J. C. Gille, 1998: Vertically stacked temperature disturbances near the equatorial stratopause as seen in cryogenic limb array etalon spectrometer data. $J$. Geophys. Res., 103, 19 469-19 483.

Hitchman, M. H., C. B. Leovy, J. C. Gille, and P. L. Bailey, 1987: Quasi-stationary zonally asymmetric circulations in the equatorial lower mesosphere. J. Atmos. Sci., 44, 2219-2236.

Holm, D. D., J. E. Marsden, T. Ratiu, and A. Weinstein, 1985: Nonlinear stability of fluid and plasma equilibria. Phys. Rep., 123, $1-116$

Holt, M. W., and A. J. Thorpe, 1991: Localized forcing of slantwise motion at fronts. Quart. J. Roy. Meteor. Soc., 117, 943 963.

Hua, B. L., D. W. Moore, and S. Le Gentil, 1997: Inertial nonlinear equilibration of equatorial flows. J. Fluid Mech., 331, 345371.

Hunt, B. G., 1981: The maintenance of the zonal mean state of the upper atmosphere as represented in a three-dimensional general circulation model extending to $100 \mathrm{~km}$. J. Atmos. Sci., 38, 2172-2186.
Kasahara, A., 2003: The roles of the horizontal component of the Earth's angular velocity in nonhydrostatic linear models. $J$. Atmos. Sci., 60, 1085-1095.

Knox, J. A., and V. L. Harvey, 2005: Global climatology of inertial instability and Rossby wave breaking in the stratosphere. $J$. Geophys. Res., 110, D06108, doi:10.1029/2004JD005068.

Lorenz, E. N., 1967: The Nature and Theory of the General Circulation of the Atmosphere. World Meteorological Organization, $161 \mathrm{pp}$.

Mu, M., T. G. Shepherd, and K. Swanson, 1996: On nonlinear symmetric stability and the nonlinear saturation of symmetric instability. J. Atmos. Sci., 53, 2918-2923.

Ooyama, K., 1966: On the stability of the baroclinic circular vortex: A sufficient criterion for instability. J. Atmos. Sci., 23, 43-53.

Perlis, S., 1952: Theory of Matrices. Addison-Wesley, 237 pp.

Phillips, N. A., 1966: The equations of motion for a shallow rotating atmosphere and the "traditional approximation." $J$. Atmos. Sci., 23, 626-628.

Rayleigh, J. W. Strutt, Lord, 1917: On the dynamics of revolving fluids. Proc. Roy. Soc. London, A93, 148-154.

Semeniuk, K., and T. G. Shepherd, 2001: The middle-atmosphere Hadley circulation and equatorial inertial adjustment. $J$. Atmos. Sci., 58, 3077-3096.

Shepherd, T. G., 1990: Symmetries, conservation laws, and Hamiltonian structure in geophysical fluid dynamics. Advances in Geophysics, Vol. 32, Academic Press, 287-338.

Shutts, G. J., and M. J. P. Cullen, 1987: Parcel stability and its relation to semigeostrophic theory. J. Atmos. Sci., 44, 13181330.

Stevens, D. E., 1983: On symmetric stability and instability of zonal mean flows near the equator. J. Atmos. Sci., 40, 882893.

Sun, W.-Y., 1995: Unsymmetrical symmetric instability. Quart. J. Roy. Meteor. Soc., 121, 419-431.

Thuburn, J., N. Wood, and A. Staniforth, 2002: Normal modes of deep atmospheres. II: $f-F$-plane geometry. Quart. J. Roy. Meteor. Soc., 128, 1793-1806.

Veronis, G., 1963: On the approximations involved in transforming the equations of motion from a spherical surface to the $\beta$-plane. I. Barotropic systems. J. Mar. Res., 21, 110-124.

_ 1968: Comments on Phillips' proposed simplification of the equations of motion for a shallow rotating atmosphere. $J$. Atmos. Sci., 25, 1154-1155.

White, A. A., 1999: Hydrostatic and quasi-hydrostatic versions of the basic "New Dynamics" equations. Met Office NWP Division Tech. Rep. 271, Bracknell, United Kingdom, 34 pp.

- and R. A. Bromley, 1995: Dynamically consistent, quasihydrostatic equations for global models with a complete representation of the Coriolis force. Quart. J. Roy. Meteor. Soc., 121, 399-418.

Zauderer, E., 1989: Partial Differential Equations of Applied Mathematics. 2nd ed. Wiley, 891 pp. 